\title{
Financiación y competitividad en la minería del cobre
}

\author{
Jorge Cruz Blanco(1), Luis de laTorre Palacios ${ }^{(2)}$ y José Antonio Espí Rodríguez ${ }^{(2)}$ \\ (1) azValor. Buy-Side Analyst. \\ jcruz@azvalor.com \\ (2) Universidad Politécnica de Madrid. \\ lutorpac@yahoo.es
}

\begin{abstract}
RESUMEN
El análisis de un proyecto de inversión en minería requiere, no sólo del conocimiento físico o geológico del mismo (que constituye la primera fase del proyecto) sino que también necesita de las condiciones del entorno económico en donde se desenvuelve. La volatilidad de los precios de los metales en el tiempo, hace que el rigor del análisis sea algo fundamental, y en él, se introducirán, tanto los condicionantes físicos o geológicos que van a caracterizar las posibilidades de aplicación tecnológica, como de un estudio muy cuidadoso de las variables económico-financieras que caracteriza la posición competitiva del proyecto de la empresa minera. Como ejemplo de todo esto se presenta un caso de inversión en una de las principales compañías productoras de cobre del mundo, Antofagasta PLC y se describen las bases del análisis efectuado con el fin de conseguir resultados eficientes.
\end{abstract}

Palabras clave: cobre, economía de minerales, financiación metales, suministro metales.

\section{Financing and competitiveness in copper mining}

\begin{abstract}
The analysis of an investment project in mining requires not only the physical or geological knowledge of the project (the first phase of the project) but also the economic conditions where it operates. The price volatility of metals prices makes the rigour of the analysis fundamental, and both physical and geological conditions should be included in the analysis that will characterize the possibilities of technological application, as well as a very careful study of the economic-financial variables that characterize the competitive position of the project and the mining company. As an example, we present an investment case in one of the main world copper producers, Antofagasta PLC, and describe the bases of the analysis carried out in order to achieve efficient results.
\end{abstract}

Keywords: copper, mineral economy, metal financing, metals supply.

\section{ABRIDGED ENGLISH VERSION}

\section{Introduction}

The price of metals such as copper participating in specific world commodity exchanges in different parts of the world is affected by offer and demand conditions with an uncertain evolution. This prevents predicting its value with the desired precision, not only at the moment of forecasting the returns from the previous feasibility study, but even after all the stages of the life cycle of the mining project. Previous mining experience should also be used, depending on the suitability of the method to the body geometry, the effectiveness and knowledge of the specialized personnel, the tight mechanical methods to be used, the good design of the necessary development of mining activity, etc. In addition, the adequacy of the metallurgical process chosen is decisive, based on the mineral characterization and its physical and chemical conditions, which in turn is based on the specific sampling previously carried out. 
Jorge Cruz Blanco, et al., 2019. Financiación y competitividad en la minería del cobre. Boletín Geológico y Minero, 130 (1): $81-98$

\section{Geology, technology and economics}

In a mining investment project, it is crucial to take into account the existence of a long maturation period referring to the technical concepts to be taken into account by an investor when approaching the understanding of the complexity of the mining project process. Expressed in the form of an evolution curve, the place named as "Orphan Period" in Figure 1, is the critical moment when the company, having demonstrated the existence of ore and the viability of its extraction, will need to obtain the necessary funds to build and start the project. One of the main pitfalls that investors suffered in the last century was confidence in the base reserves of the interested site, not only for regarding the good work of the explorer company, but also its sincerity. This has been alleviated to some extent by the appearance of various standards (JORC, VALMIN, NI 43-101 and others)

In a mining project it is difficult to find a technical or geological concept that does not reflect the economic aspects. The adequate geological knowledge of the mineral deposit is related with indispensable recognition by means of drill holes spaced with the minimum distance to guarantee the ore continuity necessary for an acceptable level of confidence, not only with respect to the geometry of the deposit, but also to the mineral grade. The variations in the ore grade (average, statistical) over the cut-off grade (limit, covering the direct costs) will involve the economic operating margin. When proposing an investment, the knowledge of the different steps that must be fulfilled by a mining company is essential: the elaboration of the preliminary economic assessment (PEA), the preliminary feasibility assessment (PFS) a technical report NI 43-101, a basic, detailed and economic engineering warranty, as well as the use of a good strategy for the appropriate control of critical paths.

Financing new projects in the world of copper mining

The "Orphan Period" is the moment in the life of the project where the main capital contribution is necessary to cover the construction and startup needs of the operation. For this, there are different types of financing:

1. Seed money, IPO, private placement: in this type of financing, the funds go directly into cash and equity, so there are no financial commitments with third parties, nor "covenants" as financial development restrictions. In this way they improve the balance sheet ratios, enabling greater indebtedness, without carrying any associated risk.

2. Debt, interest and maturities: they involve a low capital cost, without diluting the value of the project, or the action and with the advantage that the financial costs are tax deductible.

3. Mixed, equity and debt. Convertibles and options: combine the advantages and disadvantages of debt and "equity."They do not have the right to vote if they do not convert into shares. They tend to be "small" financing, since the value of the instrument is very volatile and the ordinary shareholder loses priority.

4. Joint ventures and project financing: means the appearance of a new partner that usually contributes a significant "know how" and that will have to perform the corresponding "due diligence", giving greater credibility to the project and producing a "de-risk" immediate to it.

5. Royalties / streaming: an expert performs a "due diligence" and purchases a part of the production in advance (the royalty). In this way, the expert buys an amount at a closed price ("streaming").

6. Operative cash flow: with this figure, the company is financially autonomous with its project, without affecting the capacity of indebtedness.

The analysis of supply and demand in the world of copper

In order to achieve the most opportune investment, in mining it is necessary to carry out an analysis of supply and demand, as directors of prices in the market, and which incorporates all the shareholders involved in the life cycle of the mining project: producers, consumers, governments, society, organizations, as well as fixing the moment in which it is within the general economic cycle.

Competitive business position. Cost curve

The cost or competitive position curve is a very practical tool that shows in an orderly manner the costs incurred by the different mining companies in obtaining one ton of copper (USD/t or cUSD/lb) per operation of a mine or centre project. In this way, the investor can get an idea, for example, about the risk that would be incurred by investing in a company with operating costs very close to the copper spot price. The graph in Figure 5 shows the existence of mining projects located above the metal-sale price line, indicating that all the projects whose cash costs exceed this line are found to have no economic viability. 
It is usual to introduce grade-tonnage curves in the standardized technical reports on mineral resources and reserves (Fig.8). These curves are constructed from the "block model" and produce an interesting view of the real possibilities of a deposit introducing variations in the conditions of metal prices. Both the curves and their expression in tables are often referred to as Project Sensitivity Analysis

Behind each consolidated ratio there are a series of root causes that, through analysis, can be discovered. This knowledge allows investors to invest in real opportunities that are accompanied by a motive rather than a simple circumstantial improvement in cost (Fig. 10). Those underlying reasons, such as a mining grade drop, a labour dispute, a temporary revocation of the permit, and others, should be previously analyzed.

\section{Example of investment in copper mining: the case of Antofagasta 2016}

Antofagasta PLC has been selected as one of the main copper miners in the world, with all its operations located in Chile. In its favour, it also has an enviable competitive position, since it has a low-cost position (located in the area below the 50th percentile), which is optimal with respect to the risk of falling copper prices (Fig. 11).

During the analysis of the example, the price of copper was in a decline of more than $50 \%$ from its maximum. Thus, copper was at its minimum price for the last 30 years as shown in Figure 12 and Antofagasta's stock at a minimum, by almost $70 \%$ (Fig. 13). The company was sufficiently analyzed and fitted with the investment style pursued by the investment company, which normally does not seek dividends, being more interested in the long-term value. In addition, margins were also at minimum levels with respect to the copper industry, which, after the analysis, made it a good investment opportunity (Fig. 14).

After the analysis, the conclusions reached by the investment company were as follow:

- With copper prices for the period analyzed, $20 \%$ of world production did not cover its costs, so the destruction of supply under these conditions was total (Fig. 15). Therefore, the price of copper should rise in the coming years.

- Copper is a metal with an important exposure to consumption and, therefore, to the global growth of wealth and the progressive incorporation of the world population to higher social levels. Therefore, its demand will continue in the short and medium term.

- Antofagasta PLC is an efficient mining company, well managed, without debt, and currently it is trading well below the replacement value of its assets.

Therefore, after analyzing all of the above, Antofagasta turned out to be a company with a growth in the share valuation of $44 \%$ in 2016 and $150 \%$ since January of the same year (Fig. 16). The study determined successive purchase orders of shares in 2015, in minimum values, and its successive sale already entered the upward cycle, in 2017, with a tendency to continue rising.

\section{Conclusions}

In the analysis of an investment in mineral assets, knowledge of the environment in which your activity moves can be key to the success of the operation in the short and medium term. Thus, the direction of the evolution of metal prices should be based on a good knowledge of the factors that condition them.

As an example, mining projects rely on a reality, on a geological principle that can be summarized as the classification by genetic models. However, the sense of the model, without realizing it, comes to reflect, not only the genesis of the deposit, but also that it assumes its economic, technical and productive sense. Thus, as an example, a copper porphyry, like most Antofagasta mining projects, when accepting the term, also accepts its generic conditions of a very low open-pit waste/ore ratio, a more or less spheroidal form, low grade, knowledge or metallurgical domain of its concentration, metal grade interval of its concentrates and several other aspects.

To close the cycle and as reflected in the previous example, the reality of the environment of the project is framed in the knowledge of both the physical factors and the economic-financial envelope that accompanies the mining project.

\section{Introducción}

Cuando una empresa de inversión se plantea acometer un proyecto dentro de un sector de la complejidad del de la minería, resulta prioritario dominar el cono- cimiento del amplio entorno dónde se desenvolverá la operación. Esto incluso antes del estudio económico con las referencias a sus márgenes de ganancias. El beneficio económico de un proyecto de explotación minera, que es el interés principal de sus accio- 
nistas desde la visión económica clásica, viene determinado, principalmente, por la cantidad de metal o mineral obtenido tras su extracción y tratamiento metalúrgico, a su precio de mercado.

El precio de metales como el cobre, que participa en bolsas mundiales específicas de materias primas en diferentes partes del mundo, viene marcado por una oferta y demanda con una evolución en todo modo incierta. Esto impide predecir su valor con la precisión deseada, no ya sólo en la hora temprana de prever las rentabilidades desde el estudio previo de viabilidad, sino incluso, superadas todas las etapas del ciclo de vida del proyecto minero (J.A. Espí y L. de la Torre, 2017)

Respecto la determinación de la cantidad total de metal o mineral a producir, ésta vendrá condicionada por la geología del yacimiento, permitiendo un estudio adecuado de sus recursos o reservas, así como de su tipología o modelo de formación geológica. El conocimiento de la geología resulta crucial si se pretende poseer la información necesaria en el medio plazo para manejarse, a nivel global, dentro de un mercado tan volátil como el del cobre.

También, se deberá utilizar la experiencia minera previa, en función de la adecuación del método a la geometría del yacimiento, de la efectividad y conocimiento del personal a contratar, de la eficacia de los métodos mecánicos a utilizar, del buen diseño de las necesarias para el desarrollo de la actividad minera, etc. Además, resulta decisiva la adecuación del proceso metalúrgico elegido, en función de la caracterización mineral y sus condiciones físicas y químicas, a partir del muestreo específico realizado previamente.

\section{Generalidades técnicas y económicas}

Resulta clave comprender con el suficiente detalle los conceptos técnicos y económicos que rodean a la actividad extractiva, a fin de poder analizar correctamente un proyecto minero, a la hora de plantearse invertir en él.

Así, dentro de los conceptos técnicos a tener en cuenta por un inversor a la hora de acercarse a comprender la complejidad de un proyecto de inversión minero, resulta crucial tener en cuenta la existencia de un largo período de maduración, que necesitará de las aportaciones de capital exigidas en cada momento. Este conocimiento avisará al inversor de los diferentes momentos de riesgo por los que se aventura.

Expresado en forma de curva de evolución, el lugar denominado "Orphan Period" en la Figura 1, resultará el momento crítico donde la empresa, una vez demostrada la existencia de mineral y la viabilidad de su extracción, necesitará conseguir los fondos necesarios para construir y arrancar el proyecto.

El ciclo de vida de una explotación minera comien-

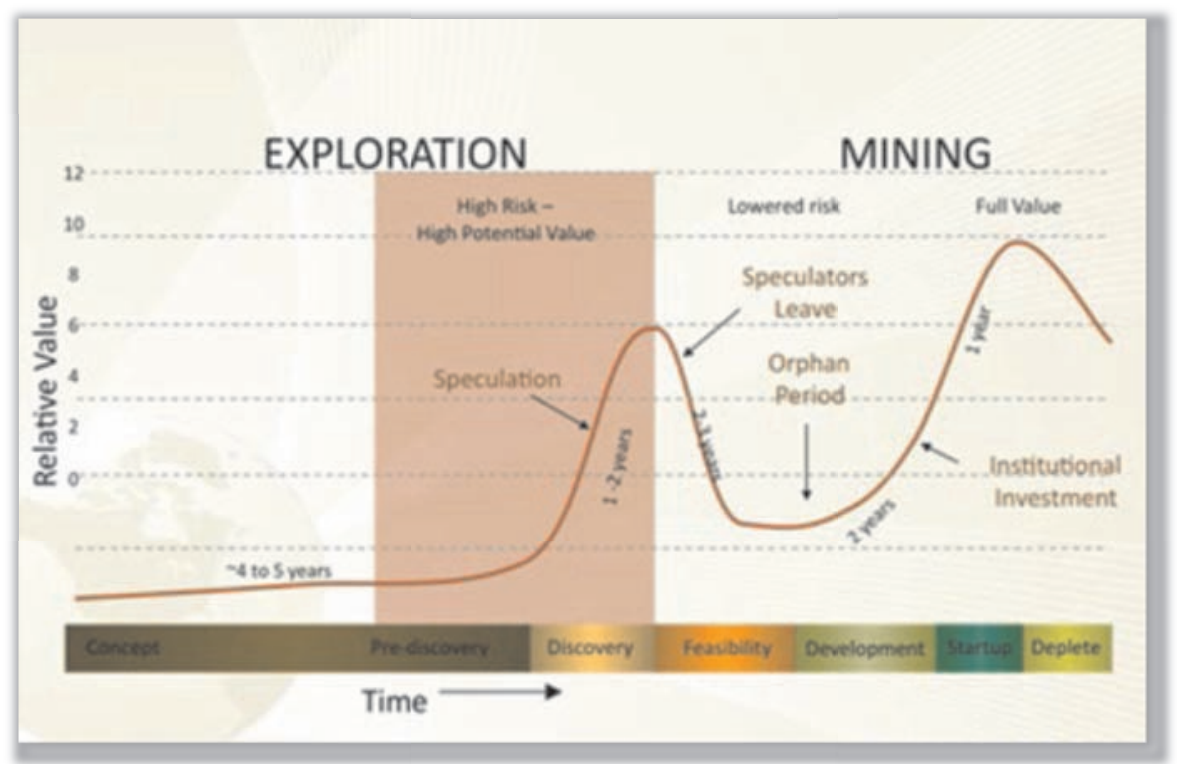

Figura 1. Fases en el desarrollo de un proyecto de explotación minera y su creación de valor (Exploration Insights, 2016) https://www.explorationinsights.com/articles.

Figure 1. Phases in development of mining projects and its value creation (Exploration Insights, 2016) https://www.explorationinsights. com/articles. 
za con la fase de exploración, pre-descubrimiento y descubrimiento de los recursos minerales, con los consecuentes estudios de ingeniería y de factibilidad económica. La definición de reservas minerales resulta clave para entender la capacidad de generar beneficios, por lo que se deberán seguir alguno de los estándares establecidos para su aceptación internacional, a la hora de acceder a la inversión. El desarroIlo constructivo del proyecto minero y el arranque de la mina (start-up) son todavía momentos de gran incertidumbre. Las fases de desinversión y el cierre junto con la etapa de su restauración, deberán estar contempladas siempre en el proyecto inicial, a fin de que no aparezcan después costes imprevistos que falsearían el estudio de inversión.

Resulta imprescindible para el inversor, conocer el momento exacto en que se encuentra el proyecto minero, ya que de esta manera se conocerá, no únicamente cuándo el proyecto comenzará a generar beneficios, sino también la duración de los mismos, y en función del momento en la vida de la misma, la progresión o retroceso de la cantidad y calidad del metal a obtener.

Uno de los principales escollos con el que se encontraban los inversores en el siglo pasado era la confianza en las reservas base del yacimiento interesado, no sólo por el buen hacer de la empresa exploradora, sino por la sinceridad de la misma. A raíz del escándalo Bre-X, aparecieron diversos estándares (JORC, VALMIN, NI 43-101...)

El adecuado conocimiento geológico del depósito mineral, pasa por el reconocimiento imprescindible mediante una campaña de sondeos espaciados con la mínima distancia para garantizar la continuidad de mineral atravesado y la necesaria para un aceptable nivel de confianza, no sólo respecto a la geometría del yacimiento, sino también a la riqueza del mineral (ley) a lo largo del mismo. Las variaciones en la ley del mineral (media, estadística) sobre la ley de corte (límite, que cubra los costes directos) supondrán, en principio, el margen operativo económico.

Desde una óptica operativa, resulta obligado a la hora de plantear una inversión, el conocimiento de los diferentes pasos que deben ser cumplidos por una empresa minera para que un proyecto pueda ser tomado en consideración: la elaboración del PEA (Preliminary Economic Assessment), el PFS (Preliminary Feasibility Assessment), un Technical Report NI 43-101, una ingeniería básica, de detalle y económica de garantía, así como el planteamiento de una buena estrategia para el control apropiado de los caminos críticos.

La otra visión, la esencialmente económica, debe tener en cuenta los factores macroeconómicos, entre los que se encuentran los ciclos de la economía. Con ellos se deberá intentar interpretar las tendencias en la búsqueda de la sostenibilidad de los precios o la elección de los momentos óptimos de inversión, tal como el aparecido tras la crisis financiera de 2008, el propio ciclo de las cotizaciones del metal y los razonamientos y modelos que sirven para anticipar las posibles variaciones acentuadas de oferta o demanda que marcan los precios.

No se debe olvidar, el habitual conocimiento del funcionamiento de los mercados financieros (entendiendo las variaciones de cotización de los metales en bolsa, las operaciones de cobertura de los precios de producción, etc.). Respecto a los factores financieros específicos y dependiendo del momento de la inversión, la empresa propietaria del proyecto minero, deberá analizar el estado de la Cuenta de Resultados, la composición del Balance (su deuda) y el estado de los Flujos de Caja. Además, en el proyecto en concreto, se observarán suTIR y VAN, sus costes operativos, el alcance de la inversión y la manera en que está financiado el proyecto.

Resulta difícil encontrar un concepto técnico o geológico de peso que no tenga reflejo en los aspectos económicos. Por ejemplo, en función de la elección del método de explotación minera, aparecerán unos costes operativos ("OPEX") diferentes, otras leyes o riquezas medias y unos subproductos más o menos valiosos. Afectarán igualmente a los costes de operación, la cantidad de reservas que marcará los años de operación y, por tanto, influirá en el VAN del proyecto el ratio de desmonte $y$, además, el desmonte inicial que puede intervenir en la inversión, al igual que el coste del cierre de la mina que figurará como provisión.

\section{Financiación de nuevos proyectos en el mundo de la minería del cobre}

Aunque el inversor puede decidir entrar a formar parte del proyecto en cualquier momento de la vida del proyecto, desde la compra del derecho minero hasta la compra de una operación minera en funcionamiento, el "Orphan Period" es el momento en la vida del mismo donde es necesario el principal aporte de capital para cubrir las necesidades de construcción y arranque de la explotación. Para esto, existen diferentes tipos de financiación, con sus ventajas e inconvenientes que permiten aplicar los fondos, desde el inversor, hasta la empresa encargada de su explotación y puesta en mercado. Habitualmente serían los siguientes:

1. Seed Money, IPO, Private Placement: en este 
Jorge Cruz Blanco, et al., 2019. Financiación y competitividad en la minería del cobre. Boletín Geológico y Minero, 130 (1): $81-98$

tipo de financiación, los fondos entran directamente a caja y a patrimonio, por lo que no hay compromisos financieros con terceros, ni "covenants" como restricciones de desarrollo financiero. De esta manera mejoran los ratios del balance, habilitando un mayor endeudamiento, sin llevar ningún riesgo asociado. Sin embargo, estos sistemas conllevan un efecto diluyente del valor de la compañía para los accionistas previos, resultando un efecto destructivo del valor cuando se aplica recurrentemente.

2. Deuda, intereses y vencimientos: suponen un bajo coste de capital, sin diluir el valor del proyecto, ni de la acción, y con la ventaja de que los costes financieros son deducibles de impuestos. Existe la posibilidad, en una nueva negociación de refinanciar las condiciones iniciales. El principal inconveniente es la existencia de un compromiso financiero con terceros, lo que puede significar la existencia de "covenants", siendo la empresa quien asume el riesgo. En caso de impago ("default"), los bonistas o los bancos se pueden quedar con la empresa, con prioridad ante los accionistas.

3. Mixtos, Equity y Deuda. Convertibles y Opciones: combinan las ventajas y los inconvenientes de la deuda y el "Equity". No tienen derecho a voto si no convierten en acciones. Suelen ser financiaciones "pequeñas", ya que el valor del instrumento es muy volátil y el accionista ordinario pierde la prioridad. Son instrumentos complejos de valorar, debido a su cambio total de valor en los distintos escenarios y consumen capacidad de endeudamiento.

4. Joint Ventures \& Project Financing: significa la aparición de un nuevo socio que suele aportar un significativo "know how" y que habrá de realizar la "due diligence" correspondiente, dando una mayor credibilidad al proyecto y produciéndose un "de-risk" inmediato del mismo. En este caso mejoran las condiciones de financiación con el Project Financing que, además, suele cubrir el total del proyecto (no hay mayores necesidades). Sin embargo, la ecuación de canje puede ser abusiva y puede existir una excesiva pérdida de valor a cambio de una disminución del riesgo. Suele ser una solución beneficiosa a corto plazo, pero diluyente en el largo, perdiendo de esta forma el control mayoritario del activo. Ello impide vender o llevar a cabo ciertas actividades.

5. Royalties / Streaming: un experto realiza una "due diligence" y compra una parte de la producción por adelantado durante un tiempo pactado (royalty). De esta forma, compra una cantidad a un precio cerrado ("streaming"), lo que otorga mayor credibilidad al Proyecto, pudiendo indicar, según los términos, que el Proyecto tiene más potencial del previsto inicialmente. No hay deuda financiera, por lo que no se ve afectada la capacidad de endeudamiento. Son fondos que van a caja y al patrimonio neto. La parte negativa se refiere a que las empresas de "royalties / streaming" se podría decir que actúan como "tiburones" que aprovechan las necesidades de los proyectos, pudiendo alcanzar una TIR de hasta el $200 \%$, generando habitualmente un contrato muchas veces con condiciones abusivas. Por ello, se suele emplear en cantidades pequeñas, teniendo prioridad por encima de todos los acreedores.

6. Cash Flow Operativo: con esta figura, la empresa es autónoma financieramente con su proyecto, sin afectar a la capacidad de endeudamiento. Existe cierta discusión sobre si se optimiza o no el coste del capital, al ser la deuda más barata. Sin embargo, ello significa una cierta pérdida en el control del proyecto. Dada la cuantía habitualmente a financiar, no está al alcance de cualquier proyecto. De esta manera, solo pueden proceder así las grandes empresas como Rio Tinto, Antofagasta, Southern Copper, etc. que tienen varias minas en operación. No existe auditoría por terceras partes.

Como ejemplo, en la Tabla 1, se muestra los diferentes tipos de financiación empleados por varias de las principales empresas mineras.

Para poder disponer de un orden de la magnitud de las inversiones en los principales proyectos actuales, se observa en la Tabla 2 como la cifra global de gasto actualmente puede superar los 100.000 millones de dólares, ya que en ella se muestran tan solo los proyectos que sobrepasan la increíble cantidad de 4.000 millones de dólares de inversión.

\section{Estrategias empresariales llevadas a cabo en el últi- mo ciclo del cobre}

El empleo de un ciclo por parte de un inversor se limita, no tanto a predecir el precio futuro, sino a lograr entender la evolución del mismo.

Si consideramos el ciclo correspondiente al período que transcurre desde el final de la crisis financiera de 2008, el fuerte aumento de la producción de cobre debido a la demanda china continúa con una caída posterior del precio del metal rojo (Fig. 2). Esto se debe a la disminución de la demanda de cobre y a la sobreproducción procedente de unas fuertes inversiones ante los elevados niveles de precios del metal. Esto sucede hasta la recuperación de los precios a mediados de 2016 y se pueden analizar la serie de estrategias llevadas a cabo por las principales empresas mineras del mundo del cobre. 


\begin{tabular}{|c|c|c|c|}
\hline PROYECTO & OPERADOR & FINANCIACIÓN & DATOS \\
\hline Cobre Panamá & First Quantum & Bonos + Bancos + Royalty & $\begin{array}{c}\text { Panamá } \\
\text { Capex: } 6.000 \mathrm{~mm} \$ \\
\text { Producción: } 300 \mathrm{ktpa} \\
\end{array}$ \\
\hline Oyu Tolgoi & Rio Tinto & Cash Flow Operativo & $\begin{array}{c}\text { Mongolia } \\
\text { Capex: } 4.200 \mathrm{~mm} \$ \\
\text { Producción: }+280 \mathrm{ktpa} \\
\end{array}$ \\
\hline Antucoya & Antofagasta & Cash Flow Operativo & $\begin{array}{c}\text { Chile } \\
\text { Capex: } 1.500 \mathrm{~mm} \$ \\
\text { Producción: } 85 \mathrm{ktpa}\end{array}$ \\
\hline Buenavista & Southern Copper & Cash Flow Operativo + Deuda & $\begin{array}{c}\text { México } \\
\text { Capex: } 3.500 \mathrm{~mm} \$ \\
\text { Producción: }+300 \mathrm{ktpa} \\
\end{array}$ \\
\hline Cerro Verde & Freeport & Deuda + Project Financing & $\begin{array}{c}\text { Perú } \\
\text { Capex: } 4.600 \mathrm{~mm} \$ \\
\text { Producción: }+275 \mathrm{ktpa} \\
\end{array}$ \\
\hline Kamoa-Kakula & Ivanhoe & Equity + Project Financing & $\begin{array}{c}\text { DR Congo } \\
\text { Capex: } 1.200 \mathrm{~mm} \$ \\
\text { Producción: } 100 \mathrm{ktpa}\end{array}$ \\
\hline
\end{tabular}

Tabla 1. Proyectos recientes y su financiación.

Table 1. Actual projects and their financing.

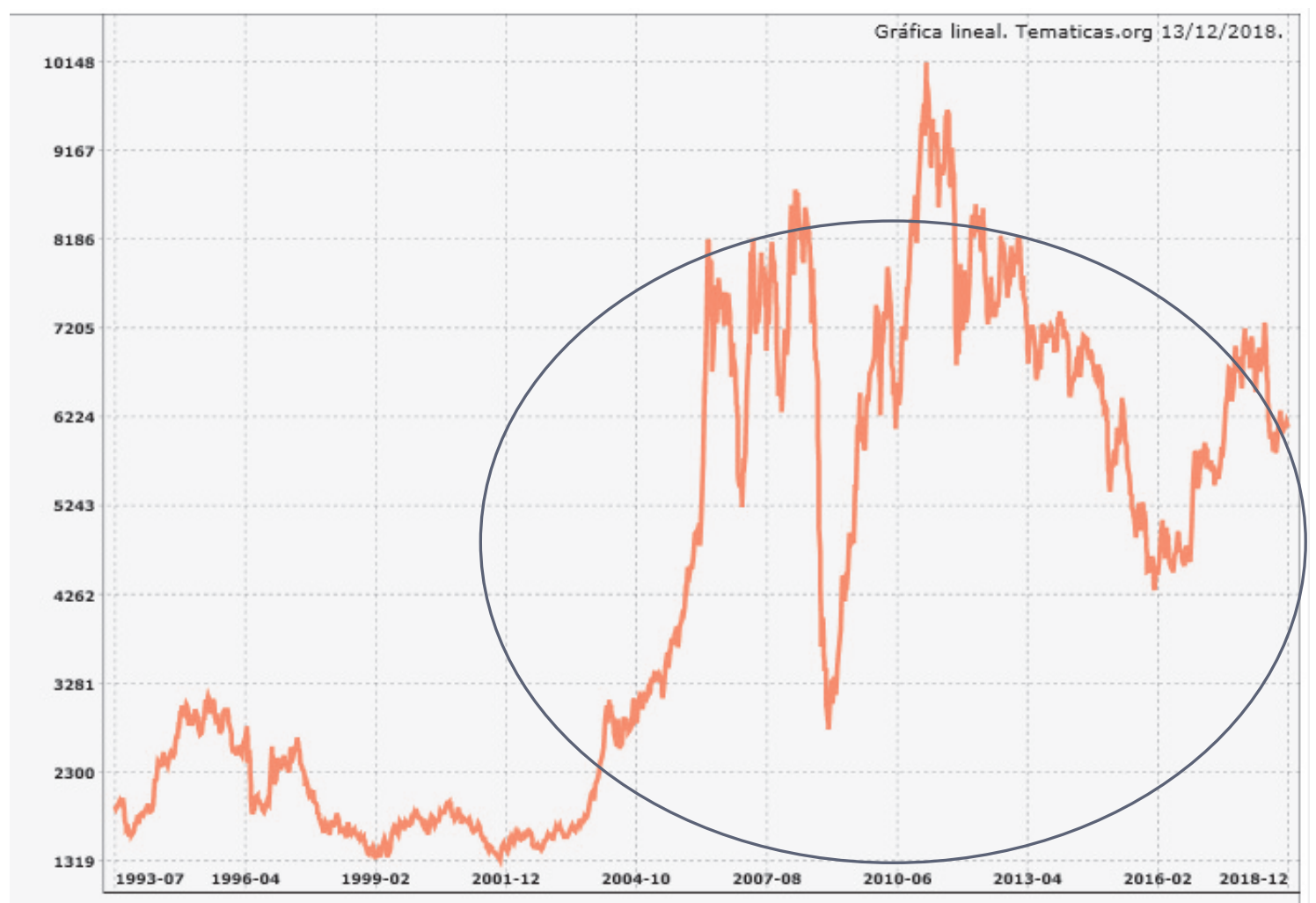

Figura 2. Evolución de los precios del cobre. Ciclo analizado. (según tematicas.org. 2018).

Figure 2. Evolution of copper prices. Cycle analyzed. (tematicas.org. 2018). 
Jorge Cruz Blanco, et al., 2019. Financiación y competitividad en la minería del cobre. Boletín Geológico y Minero, 130 (1): 81-98

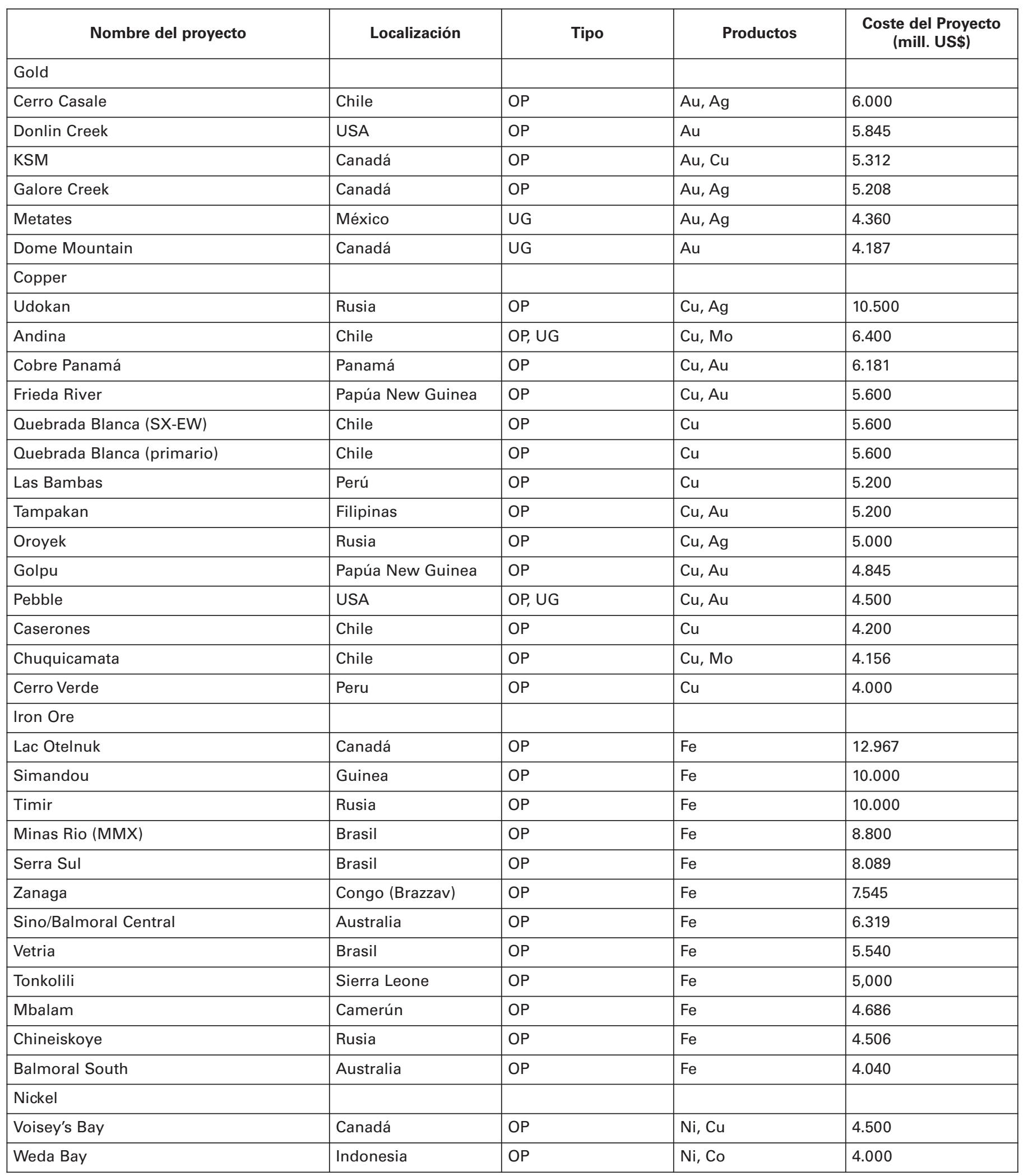

Tabla 2. Coste de los principales proyectos en la minería del cobre hasta 2015 (según E\&MJ's Annual Survey of Global Metal-mining Investment). Tipo de minería: PO cielo abierto; UG subterránea.

Table 2. Cost of the main projects in copper mining up to 2015 (E\&MJ's Annual Survey of Global Metal-mining Investment). Mining type: $P O$ open pit; UG underground. 
Jorge Cruz Blanco, et al., 2019. Financiación y competitividad en la minería del cobre. Boletín Geológico y Minero, 130 (1): $81-98$

\section{Actuaciones acometidas durante un ciclo alcista}

Un escenario mantenido de alza en los precios, provoca que la industria acepte éstos y los considere sostenibles, al entender el inversor que los "precios spot" son reales. También ve la oportunidad de obtener grandes beneficios con ellos, generando un importante incentivo para nuevas inversiones, apareciendo un gran número de proyectos que resultan rentables, cuando unos meses atrás no lo eran.

En esos momentos, gran parte de las inversiones se realizan con deuda, con unas bajadas del coste de capital que las convierten en aún más atractivas. Haciendo un símil, se podría recordar el "boom" inmobiliario, con intereses muy bajos y abundancia de dinero que invertir. Las empresas operadoras ganan más dinero del habitual, con lo que se ven en la "obligación" de invertir esos beneficios, además del reparto de dividendos. También se producen efectos multiplicativos, debidos a los altos precios, previendo los mercados financieros un mantenimiento en los precios, o incluso esperando mayores subidas. Ello potencia la entrada de inversores financieros con compra de futuros, derivados y otros productos financieros y favorece así la especulación, además de otras distorsiones del mercado.

Esto produjo, como se observa en la Tabla 3, que en el año 2011 se superaran los 120.000 millones de inversión, que supone un $20 \%$ más de lo alcanzado en la actualidad. Este exceso de oferta, ante la disminución de la demanda, contribuyó a la bajada de los precios del cobre.

Entre las diversas estrategias empleadas por las empresas mineras durante el ciclo alcista, se encuentran las siguientes: se acometen inversiones incluso con unos CAPEX (inversiones iniciales) elevados por unidad de producción, aparece el inicio de actividad de yacimientos con altos costes operativos, e incluso llega a la explotación minera el incremento de los costes fijos (por ejemplo, en gastos de personal, en bonus, negociación con sindicatos, etc.).

La compra de activos, junto con las tres actividades mencionadas en el párrafo anterior, trae consigo, en un escenario de precios altos y la temida destrucción de valor para el accionista. Todo ello resulta en un aumento de riesgo para un posible escenario futuro de bajada de precios.

Sin embargo, una ampliación de capital aprovechando el elevado precio de la acción, o bien, una venta de activos mediante fusiones y adquisiciones (M\&A) cuando el precio está muy alto, generan valor para el accionista. Otro ejemplo de creación de valor es la inversión en exploración y eficiencia operativa, imprescindible para el mantenimiento de cualquier empresa minera. También genera valor para el accionista el emitir deuda en un momento de bonanza, aprovechando una tasa de interés mucho más baja (prima por riesgo asociado al negocio), o bien, repartir dividendos y crear reservas financieras

\section{Actuaciones acometidas durante un ciclo bajista.}

Al igual que ocurre en los momentos de alza de precios, la industria inmediatamente entiende el declive de los "precios spot" del mercado, y los toma rápidamente como una realidad sostenible en el tiempo. Los bajos precios generan aversión a la inversión, parando o retrasando las inversiones en marcha, al dejar estas de ser rentables para el inversor.

Los operadores en pérdidas las financian con deuda o "equity", prolongando su caída y no depurando en el momento los malos proyectos. A pesar de la bajada de precios, las explotaciones mineras continúan produciendo, ya que no resulta sencillo decidir una parada de producción por los costes que conlleva y lo complejo de volver a ponerlas en operación. Por ello, mientras aguanten las finanzas de la empresa, ésta continuará extrayendo mineral a pesar de perder dinero, en espera de un alza de precios en el corto plazo.

En este escenario, las empresas operadoras no ganan dinero e intentan evitar las pérdidas mediante M\&A, en búsqueda de optimizaciones operativas $y$ disminución de costes fijos. Se producirá así una crisis a escala, donde mueren los más pequeños, o aquellos que se encuentren en la curva de costes entre los peor situados, y los grandes, se convierten todavía en más grandes, alcanzándose, por tanto, una mayor globalización de la industria.

En cuanto a la estrategia llevada a cabo en este escenario de ciclos bajistas, ante unos precios considerados inferiores a los reales, la principal y ya comentada, es la parada de las inversiones, al disminuir los ratios de rentabilidad elevados a los que los inversores estaban acostumbrados.

En el proyecto minero se puede cambiar el "Mining Plan", acometiendo, en caso de necesidad financiera, los frentes de mayor ley y menor ratio de desmonte. En la práctica, existen diversas tácticas de actuación, como la de guardar las mejores leyes para momentos de precios altos. La necesidad puede Ilevar a la venta de activos a bajos precios, ampliaciones ruinosas de capital, o bien, a emitir una deuda donde la penalización por el riesgo asociado dispara su coste. Ello puede significar, además de esta serie de medidas, una destrucción de valor para el accionista. 
Jorge Cruz Blanco, et al., 2019. Financiación y competitividad en la minería del cobre. Boletín Geológico y Minero, 130 (1): $81-98$

\begin{tabular}{|c|c|c|c|c|}
\hline Nombre del proyecto & Localización & Tipo minería & Productos & Inversiones en mill. \$ \\
\hline Olympic Dam & Australia & UG & $\mathrm{Cu}_{1, \mathrm{U}}$ & 8.200 \\
\hline Tampakan & Filipinas & $\mathrm{OP}$ & $\mathrm{Cu}, \mathrm{Au}$ & 5.200 \\
\hline Udokan $\mathrm{Cu}$ & Rusia & OP & $\mathrm{Cu}, \mathrm{Ag}$ & 5.000 \\
\hline Oyu Tolgoi & Mongolia & OP & $\mathrm{Cu}, \mathrm{Au}$ & 4.600 \\
\hline Pebble East & USA & OP,UG & $\mathrm{Cu}, \mathrm{Au}$ & 4.500 \\
\hline Andina & Chile & OP, UG & $\mathrm{Cu}, \mathrm{Mo}$ & 6.400 \\
\hline Cobre Panamá & Panamá & $\mathrm{OP}$ & $\mathrm{Cu}, \mathrm{Au}$ & 6.181 \\
\hline Las Bambas & Perú & $\mathrm{OP}$ & $\mathrm{Cu}$ & 5.200 \\
\hline Chuquicamata & Chile & $\mathrm{OP}$ & $\mathrm{Cu}, \mathrm{Mo}$ & 4.156 \\
\hline Escondida & Chile & $\mathrm{OP}$ & $\mathrm{Cu}, \mathrm{Au}$ & 3.800 \\
\hline ElTeniente & Chile & UG & $\mathrm{Cu}, \mathrm{Mo}$ & 3.039 \\
\hline Rekodiq & Pakistán & $\mathrm{OP}$ & $\mathrm{Cu}, \mathrm{Au}$ & 3.000 \\
\hline Resolution & USA & UG & $\mathrm{Cu}, \mathrm{Mo}$ & 3.000 \\
\hline Schaft Creek & Canadá & $\mathrm{OP}$ & $\mathrm{Cu}, \mathrm{Au}$ & 3,258 \\
\hline Aynak & Afganistán & $\mathrm{OP}$ & $\mathrm{Cu}$ & 2.968 \\
\hline Sierra Gorda & Chile & $\mathrm{OP}$ & $\mathrm{Cu}, \mathrm{Mo}$ & 3.900 \\
\hline Los Azules & Argentina & OP & $\mathrm{Cu}, \mathrm{Au}$ & 3.920 \\
\hline Haquira & Perú & OP, UG & $\mathrm{Cu}$ & 2.824 \\
\hline Cumo & USA & OP & $\mathrm{Cu}, \mathrm{Mo}$ & 2.800 \\
\hline Los Bronces & Chile & $\mathrm{OP}$ & $\mathrm{Cu}, \mathrm{Mo}$ & 2.800 \\
\hline Frieda River & Papúa N. Guinea & $\mathrm{OP}$ & $\mathrm{Cu}, \mathrm{Au}$ & 5.600 \\
\hline El Morno & Chile & OP & $\mathrm{Cu}, \mathrm{Au}$ & 2.520 \\
\hline Galeno & Perú & $\mathrm{OP}$ & $\mathrm{Cu}, \mathrm{Au}$ & 2,500 \\
\hline La Granja & Perú & OP & $\mathrm{Cu}$ & 2.500 \\
\hline Collohuasi & Perú & OP & $\mathrm{Cu}, \mathrm{Mo}$ & 2.400 \\
\hline Ministro Hales & Chile & OP & $\mathrm{Cu}$ & 3.500 \\
\hline Quellaveco & Perú & $\mathrm{OP}$ & $\mathrm{Cu}, \mathrm{Mo}$ & 2.300 \\
\hline Toromocho & Perú & $\mathrm{OP}$ & $\mathrm{Cu}, \mathrm{Mo}$ & 3.500 \\
\hline Casino Copper & Canadá & $\mathrm{OP}$ & $\mathrm{Cu}, \mathrm{Au}$ & 2.141 \\
\hline Agua Rica & Argentina & $\mathrm{OP}$ & $\mathrm{Cu}, \mathrm{Au}$ & 2.055 \\
\hline Caserones & Chile & $\mathrm{OP}$ & $\mathrm{Cu}$ & 2.000 \\
\hline El Pachón & Argentina & $\mathrm{OP}$ & $\mathrm{Cu}, \mathrm{Mo}$ & 1.900 \\
\hline Salobo & Brasil & $\mathrm{OP}$ & $\mathrm{Cu}$ & 1.808 \\
\hline Bozshakol & Kazajstán & OP & $\mathrm{Cu}, \mathrm{Au}$ & 1.800 \\
\hline El Arco & Méjico & $\mathrm{OP}$ & $\mathrm{Cu}$ & 1.700 \\
\hline Lomas Bayas & Chile & $\mathrm{OP}$ & $\mathrm{Cu}$ & 1.600 \\
\hline Canariaco Norte & Perú & & $\mathrm{Cu}, \mathrm{Au}$ & 1.565 \\
\hline
\end{tabular}

Tabla 3. Inversión en proyectos de cobre hasta el año 2011 (según E\&MJ).

Table 3. Investment in copper projects up to 2011 (E \& MJ). 
Sin embargo, existen otras actuaciones con las que las empresas intentan aguantar durante los ciclos bajistas sin destruir valor, y ellas son la disminución de costes, la revisión a la baja de los presupuestos de las inversiones, la compra de activos a bajo precio mediante M\&A, y la recompra acciones propias a precios inferiores a su valor real.

\section{¿Qué ocurre con los costes mineros cuando varían los precios?}

En comentarios anteriores, se ha mostrado como las empresas mineras del cobre pueden obtener su curva de ventas en función de la curva de precios de mercado, multiplicando por las toneladas de cobre metal producidas. El mayor interés por parte de la empresa $y$, sobre todo, para sus accionistas, es el margen obtenido, que resultará de las ventas y los costes generados en la operación.

Así, se produce el efecto "huevo-gallina", al no saber qué factor tira de cuál, si el precio a los costes, o viceversa. Obviamente, con un cobre a 10.000 US\$, las empresas no van a producir a 3.000 US\$ (aumentan los costes de los proveedores, del personal, etc.), así como tampoco resultaría creíble que una empresa realizara un proyecto de viabilidad con unos costes superiores al precio de mercado.

Sin embargo, se producen reacciones muy diferentes entre la minería a cielo abierto, donde la relación de cambio de costes resulta más inmediata (por ejemplo, la subida del precio de la energía se traduce en una elevación casi directa), y la subterránea. En la minería bajo tierra, los costes fijos y los costes de personal son significativos, y son más lentos de modificar.

\section{El análisis de la oferta y la demanda en el mundo del cobre}

Para tratar de lograr una inversión lo más oportuna posible, en minería resulta necesario realizar en primer lugar un análisis de la oferta y la demanda, como directores de los precios en el mercado que deberá incorporar a todos los actores implicados en el ciclo de vida del proyecto minero: productores, consumidores, gobiernos, sociedad, organizaciones de diversa índole, así como la fijación del momento en que se encuentre dentro del ciclo económico general y el del mineral en concreto.

En situaciones de exceso de oferta, los almacenes superan la demanda requerida en ese momento. Una vez satisfecha la demanda, el coste del último pro- ductor acordado con su cliente, será el precio del metal en ese mercado. Por otra parte, cuando la demanda supera la oferta, el precio vendrá determinado, principalmente, por el coste de la nueva unidad que entra en producción y que pone el metal en el mercado.

\section{Análisis de la demanda}

La demanda de materias primas en estos últimos años, ha venido principalmente determinada por el comportamiento de China, como principal consumidor mundial (WB, 2017). Como quedó explicado anteriormente, la mayor o menor necesidad de cobre metal por parte de la industria china ha provocado las principales variaciones en el ciclo de precios de los metales básicos.

Además, la demanda puede venir alterada por una disminución ante nuevos sustitutos, o bien, al contrario, puede incrementar su consumo gracias a la aparición de nuevos usos. Así, el vehículo eléctrico supone un claro ejemplo de nuevo uso del cobre, como oportunidad para potenciar su demanda. Mientras se continúa investigando en sustitutos para este metal (el aluminio es un gran competidor del cobre en ciertas aplicaciones, con un precio inferior a la mitad, aunque no siempre satisface todas las propiedades que ofrece el cobre), este cobre mantiene elevadas previsiones de crecimiento en su empleo en la automoción (Fig. 3), al ser necesario su uso en las baterías, en todos los motores eléctricos, en su mayor necesidad en el cableado y también, formando parte de todo aquello relacionado con el desarrollo de las nuevas energías.

Resulta comprensible que un inversor que goce de un buen conocimiento del mercado y de las necesidades industriales, donde pueda prever un incremento de la demanda ante nuevas aplicaciones que conviertan en muy valorado a un metal, aprovechará con más fortuna las oportunidades que un inversor que disponga únicamente de una formación económica.

\section{Análisis de la oferta}

El análisis de la oferta implicará el conocimiento de los diferentes productores mundiales, de sus situaciones históricas y tendencias, de sus recursos y reservas, sus costes de operación, de la estabilidad política y social que les rodea, de su buena gestión, del compromiso medioambiental existente, etc. Todos ellos son factores que afectan a la oferta de manera muy significativa, pudiendo producir un cambio ines- 


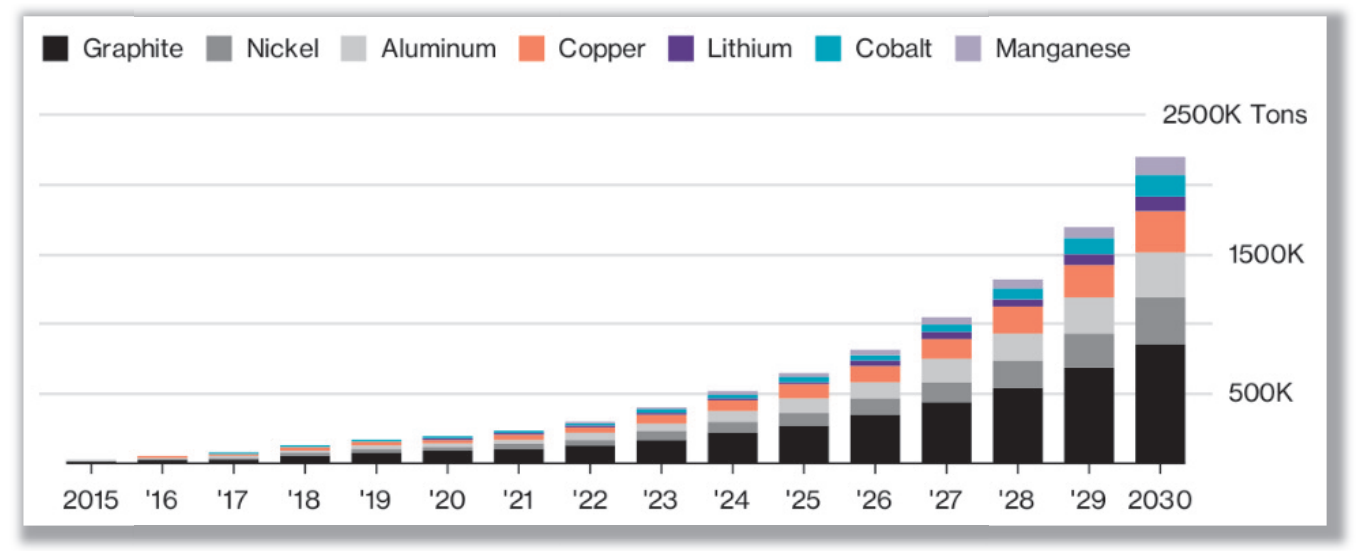

Figura 3. Incremento de la demanda de metales debido al vehículo eléctrico. (Bloomberg. 2017).

Figure 3. Increase in demand for metals due to the electric vehicle. (Bloomberg 2017).

perado en la valoración del metal en el mercado con una mínima variación en el stock de almacenes,

Chile continúa encabezando la producción mundial, seguida de Perú debido a un extraordinario crecimiento en los últimos años, seguidos ambos por China y EE.UU. En total y en el año 2016 se alcanzó a nivel mundial una producción algo inferior a los 20 millones de toneladas de cobre fino.

\section{Posición empresarial competitiva. Curva de costes}

La curva de costes o de posición competitiva, resulta una herramienta muy práctica que muestra ordenadamente los costes en que incurren las diferentes empresas mineras en la obtención de una tonelada de cobre (USD/t o c USD /lb) por explotación de un proyecto o centro minero. De esta forma, el inversor puede hacerse una idea, por ejemplo, del riesgo en que incurriría invirtiendo en una empresa con unos costes de operación muy próximos al precio spot del cobre. En la gráfica de la Figura 4 se observa la existencia de proyectos mineros situados por encima de la línea del precio de venta del metal, indicando esto que, todas las explotaciones cuyos costes de operación ("cash costs") superan esta línea, se encuentran sin sentido económico.

Igualmente se muestra la potencia de esta herramienta en la gráfica de la Figura 4, donde se observa, ya consolidados por las empresas, los costes de operación y la situación relativa a sus competidores (los otros productores), posibilitando dibujar la posición

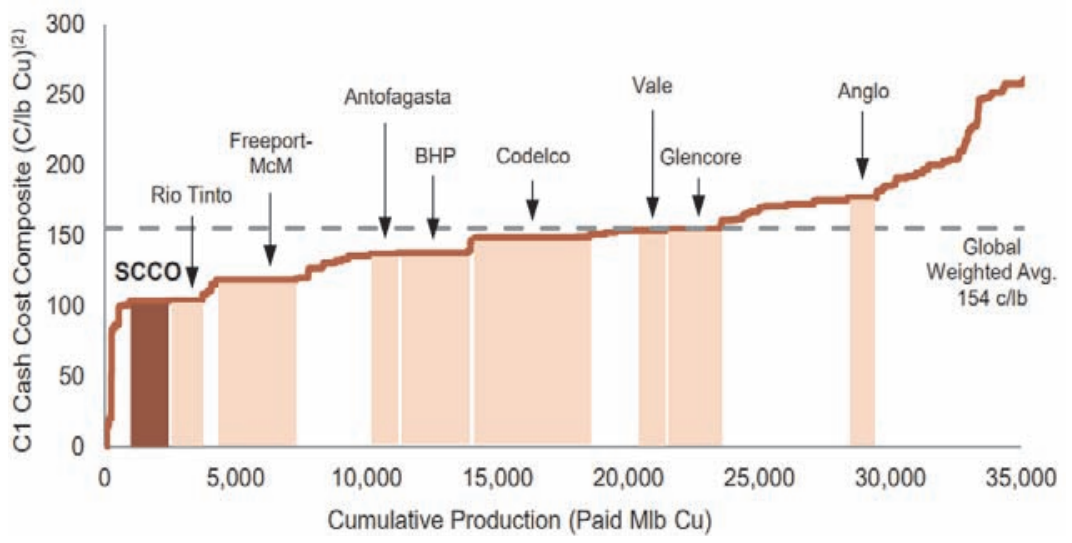

Figura 4. Curva de costes operativos respecto a una libra de cobre producida por empresas. Fuente: Southern Copper/ Wood Mackenzie. Figure 4. Curve of operating costs by companies with respect to one pound of copper produced. Source: Southern Copper/Wood Mackenzie. 
de cada empresa respecto al resto de los actores del mercado e imaginar el estilo de gestión de cada una de ellas.

Otro parámetro que influye igualmente en la oferta y por ende en los precios del metal, es el decrecimiento de las leyes de los minerales extraídos y la incorporación de yacimientos minerales de menor accesibilidad. Ambas razones presagian en un futuro próximo un aumento claro en los costes operativos e inversiones: mayor proporción en minería subterránea, explotaciones de fuentes aún no consideradas como los fondos marinos y otras situaciones.

Estudiando las relaciones entre los tonelajes acumulados por encima de distintos intervalos de leyes (leyes de corte) y dichas leyes, se observa que la calidad de la regresión entre unos valores y otros es buena (J.I. Manteca, 1993). Dicha relación es una función exponencial y está formalizada por la ley de Lasky (Wellmer F.W. and Drobe M. 2018), que da una buena aproximación al modelo lognormal y permite reproducir las relaciones entre las leyes y los correspondientes tonelajes acumulados en los depósitos minerales. Lasky estableció y ajustó esa ecuación para el conjunto de los 10 mayores pórfidos de cobre de Estados Unidos y más tarde la verificó para depósitos de sustancias muy diferentes (Fig. 5).

Actualmente es usual introducir las curvas leytonelaje en los informes técnicos estandarizados de valoración de recursos y reservas minerales (Fig.6). Estas curvas están construidas a partir del "modelo de bloques" y producen una interesante visión de las posibilidades reales de un yacimiento en el momento de introducir variaciones en las condiciones de los precios de sus metales. Tanto las curvas como su expresión en tablas se suelen denominar como Análisis de Sensibilidad del proyecto

A la hora de que un inversor quiera discernir entre la calidad de diferentes proyectos que, por ejemplo, hubieran surgido simultáneamente a causa de un incremento en el precio en el mercado, muchas veces se emplea un ratio que indica los dólares por kilotonelada de cobre fino y año de producción (Fig. 7). Como orientación, un proyecto debería estar, como inversión, entre los 12.000 USD/t y los 17.000 USD/t por año.

Detrás de cada ratio consolidado existen una serie de causas origen que, mediante el análisis, pueden ser descubiertas, permitiendo así invertir en aquellas oportunidades reales que lleven adjunto un motivo, más que una simple mejora coyuntural en el coste (Fig. 8). Esas razones subyacentes, tales como una bajada de ley, un conflicto laboral, una revocación temporal del permiso, etc. deberían ser previamente analizadas.

Otro factor a considerar son las condiciones del entorno que envuelven al país donde se encuentra el

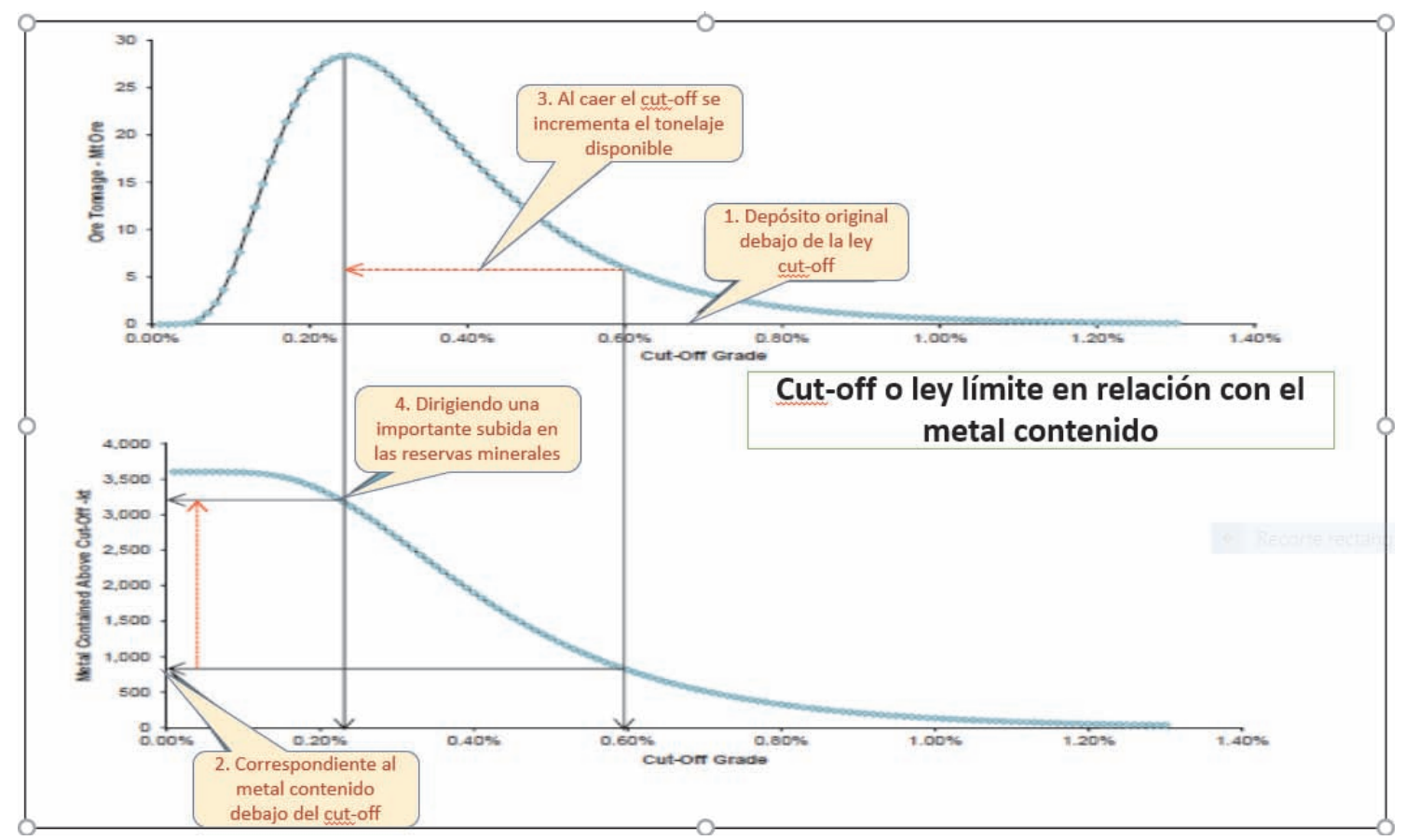

Figura 5. Relaciones entre la ley límite o "cut-off" y la ley media y el tonelaje del proyecto minero. Figure 5. Relations between the "cut-off" and the average grade and tonnage of a mining project. 


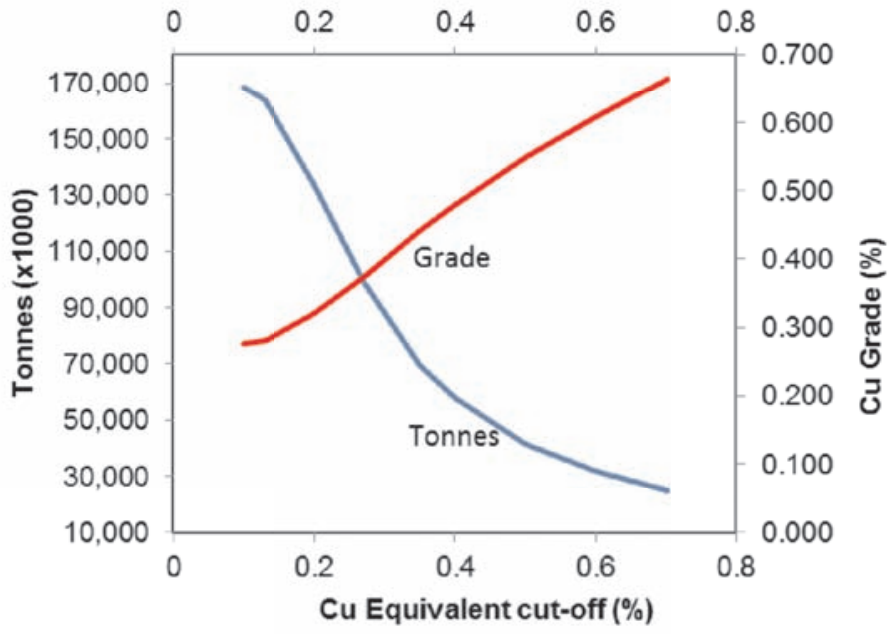

Figura 6. Curvas ley-tonelaje tomadas delTechnical Report según el estándar NI 43-101 del proyecto Kwanika Project, Serengeti Resources Inc. Año 2017.

Figure 6. Grade-tonnage curves from the Technical Report according to NI 43-101 standard of the Kwanika Project, Serengeti Resources Inc. 2017.

proyecto. Actualmente, el principal país productor de cobre, Chile, no tiene fuertes expectativas de crecimiento, y aunque Perú continúe creciendo, los países que con el tiempo podrán marcar una parte importante en la producción, serán países más conflictivos, como puedan ser Congo o Zambia, donde el inversor va a exigir unos retornos muy superiores a los actualmente esperados.

\section{Ejemplo de inversión en minería de cobre: el caso de Antofagasta 2016}

Tras el análisis llevado a cabo para evaluar las condiciones de viabilidad de una posible inversión en una compañía minera, se seleccionó a Antofagasta PLC, como una de las principales mineras de cobre del mundo, con todas sus explotaciones ubicadas en Chile, país de bajo riesgo, con amplia tradición en la minería del cobre y una regulación apropiada al tipo de actividad.

A su favor cuenta también con una envidiable posición competitiva, al encontrarse con una posición de bajo coste (situada en la zona inferior al percentil 50), que resulta óptimo respecto al riesgo de la bajada del precio del cobre (Fig. 9).

La compañía está controlada por la familia Luksic. Es una compañía sin deudas apreciables, que permite mantenerse en inversión y sin riesgo para un rápido abandono, permitiendo mantener el activo en el largo plazo, hasta que llegue la oportunidad en el mercado.

Respecto a la política de inversiones seguida por la empresa, ésta ha conseguido una buena trayectoria de asignación de capital, sin comprar nunca en los máximos del ciclo. De hecho, acababa de adquirir una mina a un vendedor 'forzado', con las ventajas que ello implica.

En el ejemplo, durante el análisis, el precio del cobre se encontraba en un descenso de más de un $50 \%$ desde sus máximos. Así, el cobre estaba en su precio mínimo de los últimos 30 años como muestra

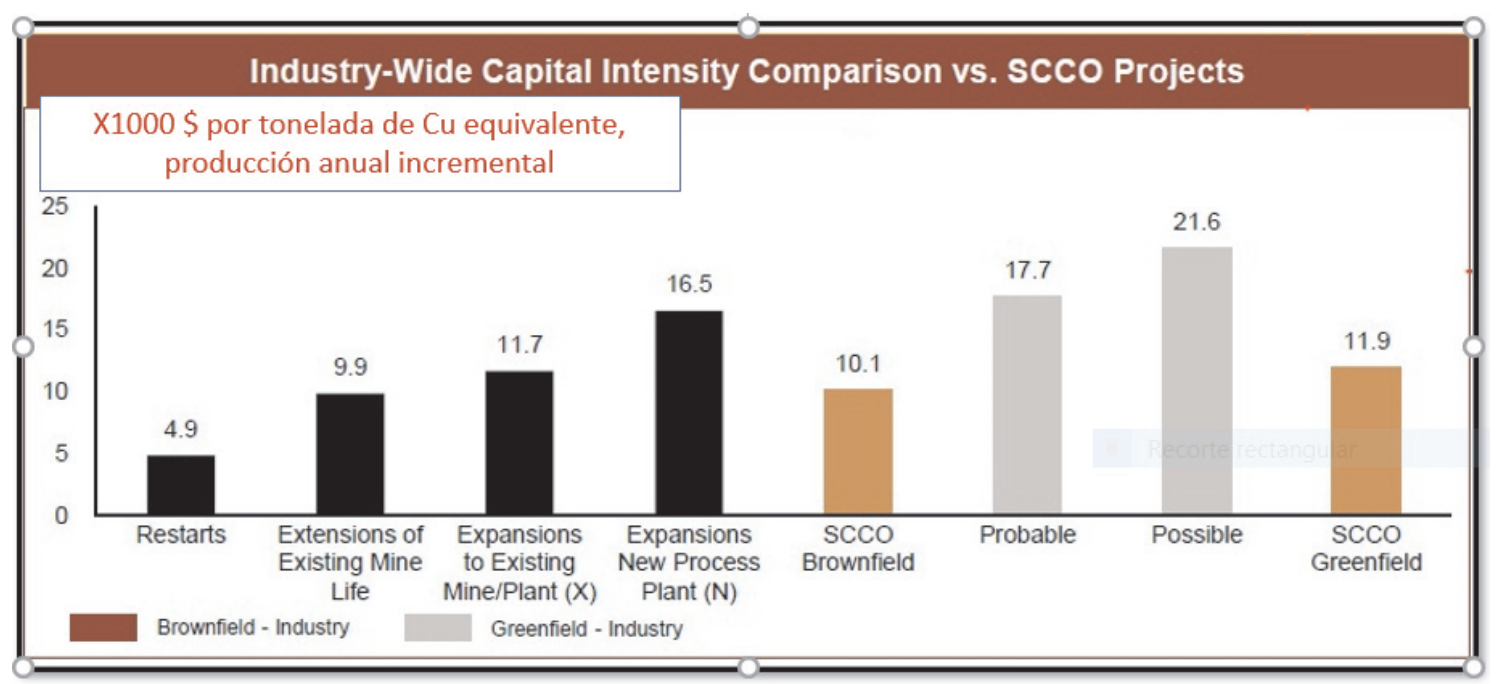

Figura 7. Ratio comparativo del capital invertido entre proyectos mineros de cobre, según Southern Copper.

Figure 7. Comparative ratio of capital invested in copper mining projects, according to Southern Copper. 


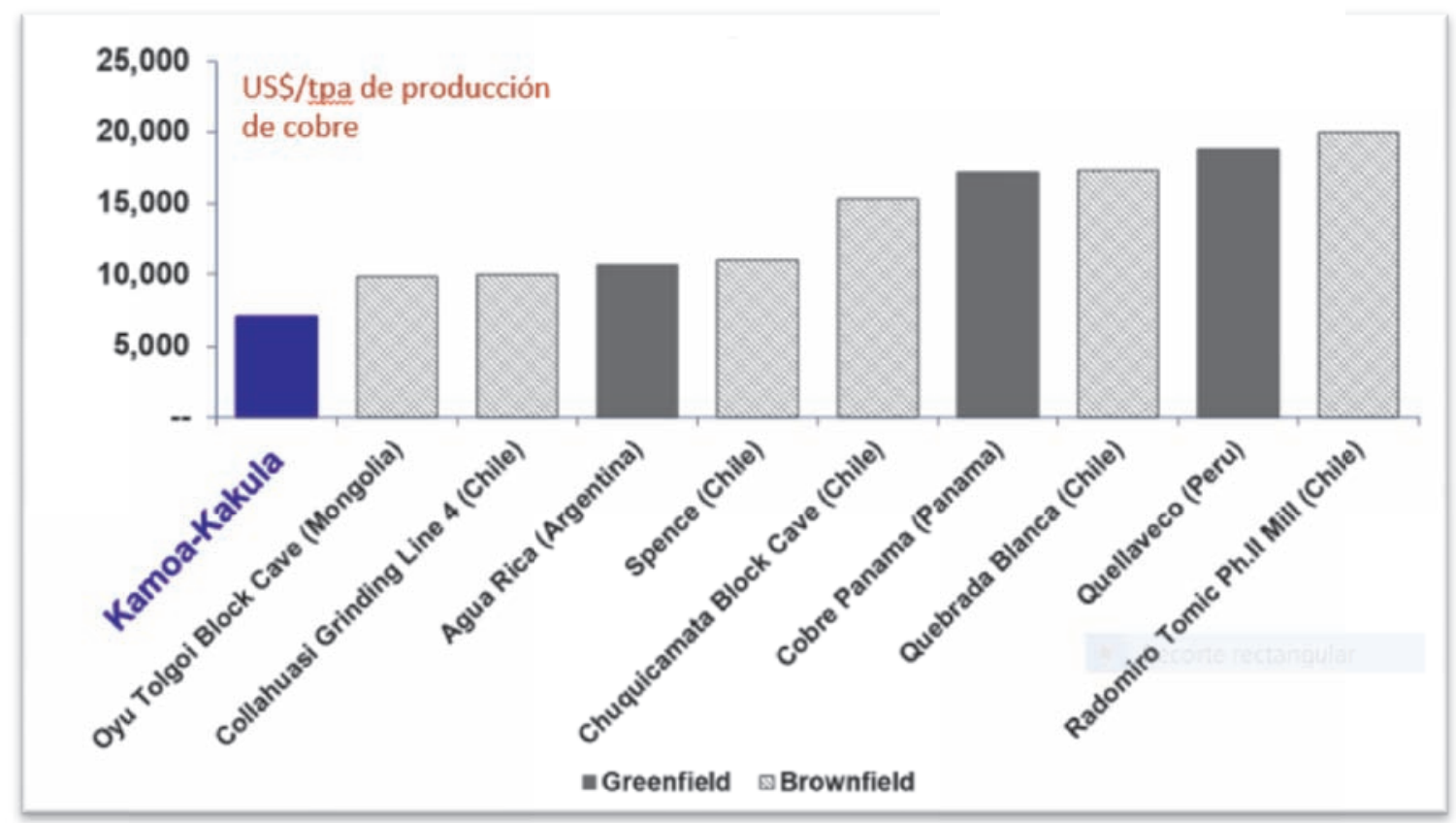

Figura 8. Intensidad del capital invertido por proyecto de cobre (Wood Mackenzie).

Figure 8. Capital intensity for large-scale copper projects (Wood Mackenzie).

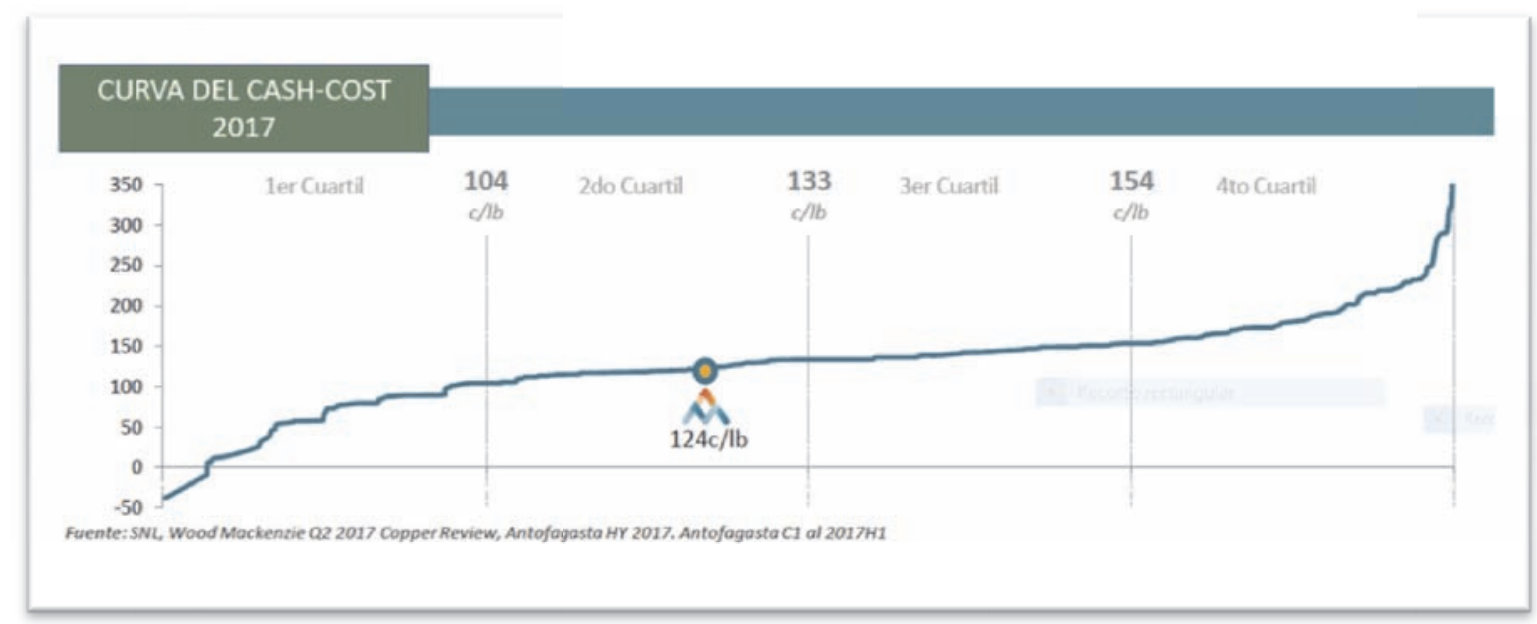

Figura 9. Coste medio del cobre metal producido en cent.\$/lb Cu por las explotaciones de Antofagasta PLC durante el año 2016. Figure 9. Average cost of copper metal produced by the copper Antofagasta PLC in cent.\$/lb Cu operations in 2016.

la Fig. 10 y el de la acción de Antofagasta en mínimos, en casi un $70 \%$ (Fig. 11).

Lo que nunca se puede conocer, es cuándo el precio del cobre va a subir, esperando una subida en los años 2016, 2017 o 2018, pero lo que sí se afirmaba tras el análisis era que la situación de precios era insostenible. Lo cierto fue que esa situación anómala se corrigió en un año, pero también cabía la posibilidad de que hubiera tardado tres o cuatro años. Ello, por otra parte, no habría sido preocupante dadas las características por las que se seleccionó el activo.
La empresa estaba suficientemente analizada y encajaba con el estilo de inversión perseguido por la empresa de inversión, que normalmente no busca el dividendo, estando más interesada en el valor a largo plazo. Además, los márgenes, también se encontraban en mínimos respecto a la industria del cobre lo que, tras el análisis, la convertía en una buena oportunidad de inversión (Fig. 12).

Tras el análisis llevado a cabo, las conclusiones a las que llegó la empresa de inversión respecto a Antofagasta fueron que: 


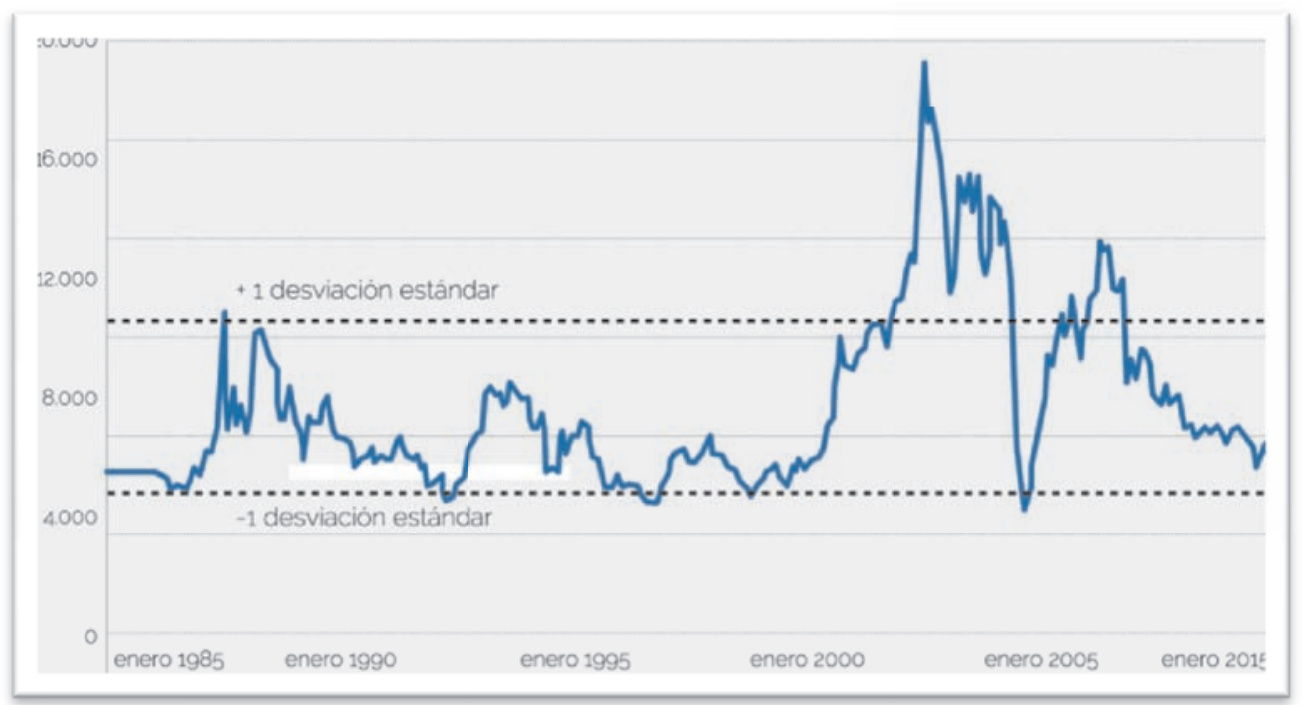

Figura 10. Precios Reales del Cobre en mínimos de 30 años (azValor).

Figure 10. Real copper prices at minimum in 30 years (azValor).

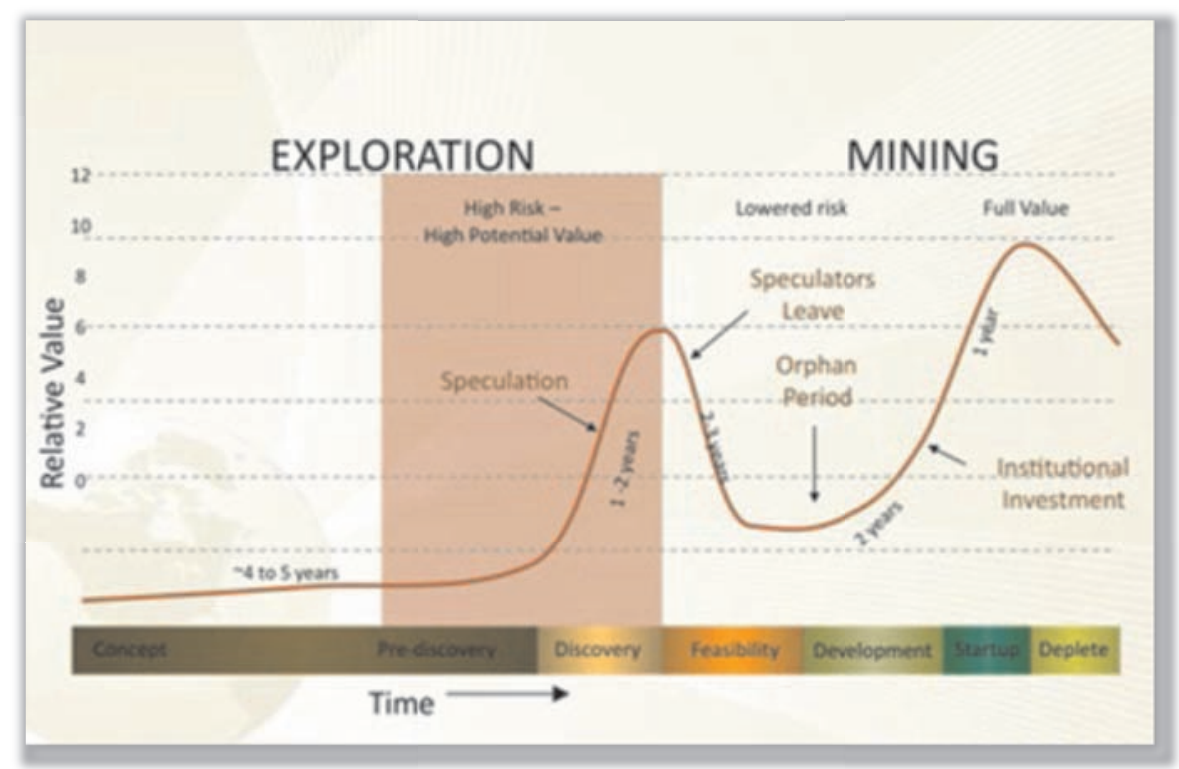

Figura 11. El mercado y Antofagasta PLC en los últimos años (azValor).

Figure 11. The market and Antofagasta in recent years (azValor).

Con precios del cobre de ese periodo, el $20 \%$ de la producción mundial no cubría sus costes. Además, la rentabilidad media en una nueva inversión generaría un ROCE inferior al 1\%. Es decir, cualquier empresa que pusiera en marcha una explotación de cobre obtendría menos del 1\%, por lo que la destrucción de la oferta en esas condiciones era total (Fig. 13). Por tanto, el precio del cobre debería subir en los próximos años.

El cobre es un metal con una importante sensibilidad al crecimiento global de la riqueza y a la incorpo- ración progresiva de la población mundial a niveles sociales más elevados. Por tanto, sin lugar a error, el cobre continuará siendo demandado en el corto y medio plazo.

Como consecuencia del gradual agotamiento de las minas a cielo abierto más superficiales, de mayor riqueza y la entrada de explotaciones subterráneas, la extracción de cobre resultará, cada vez, más costosa.

Antofagasta resulta una minera eficiente, bien gestionada, sin deuda, y que cotizaba muy por debajo del valor de reposición de sus activos. 


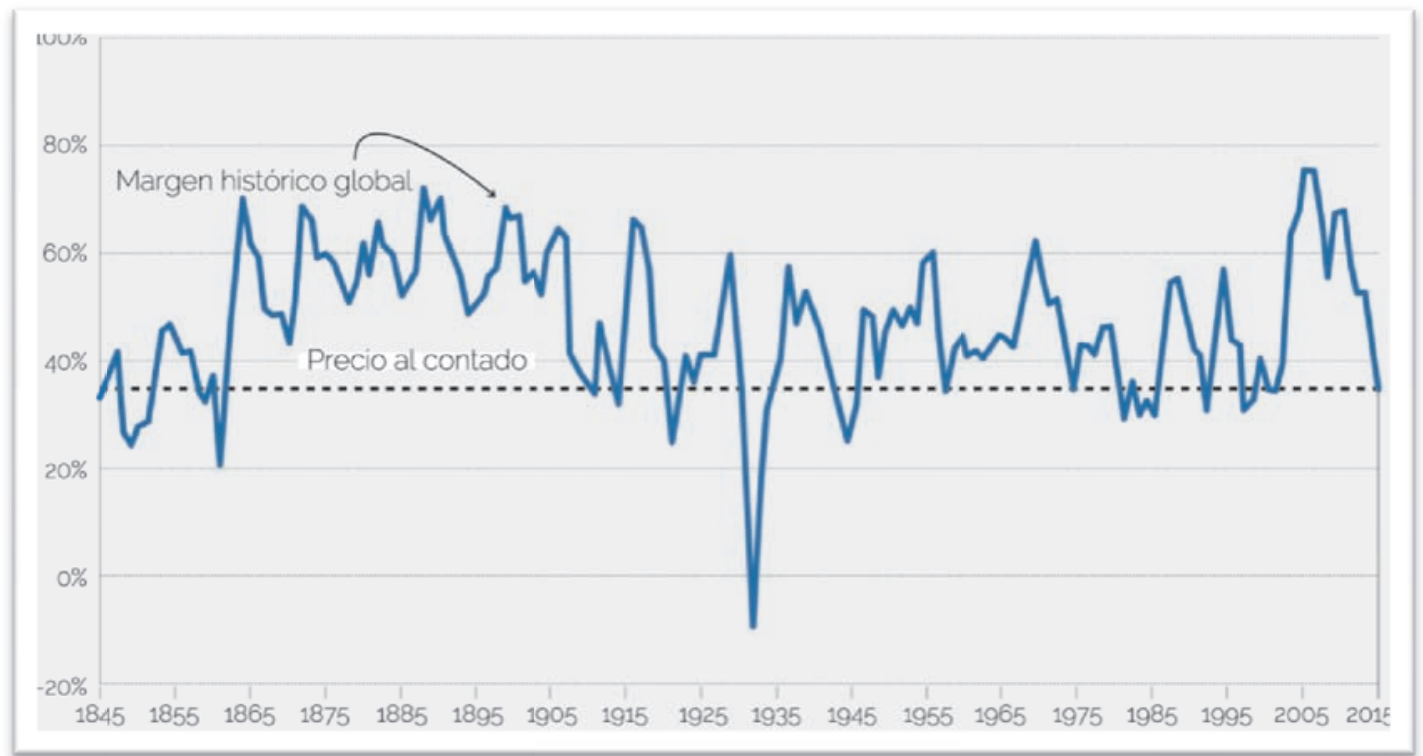

Figura 12. Márgenes mínimos desde 1845 (US Department of Commerce Bureau of Labor Statistics. Wood Mckenze y estimaciones de brokers).

Figure 12. Minimum margins since 1845 (US Department of Commerce Bureau of Labor Statistics, Wood Mckenze and broker estimates).

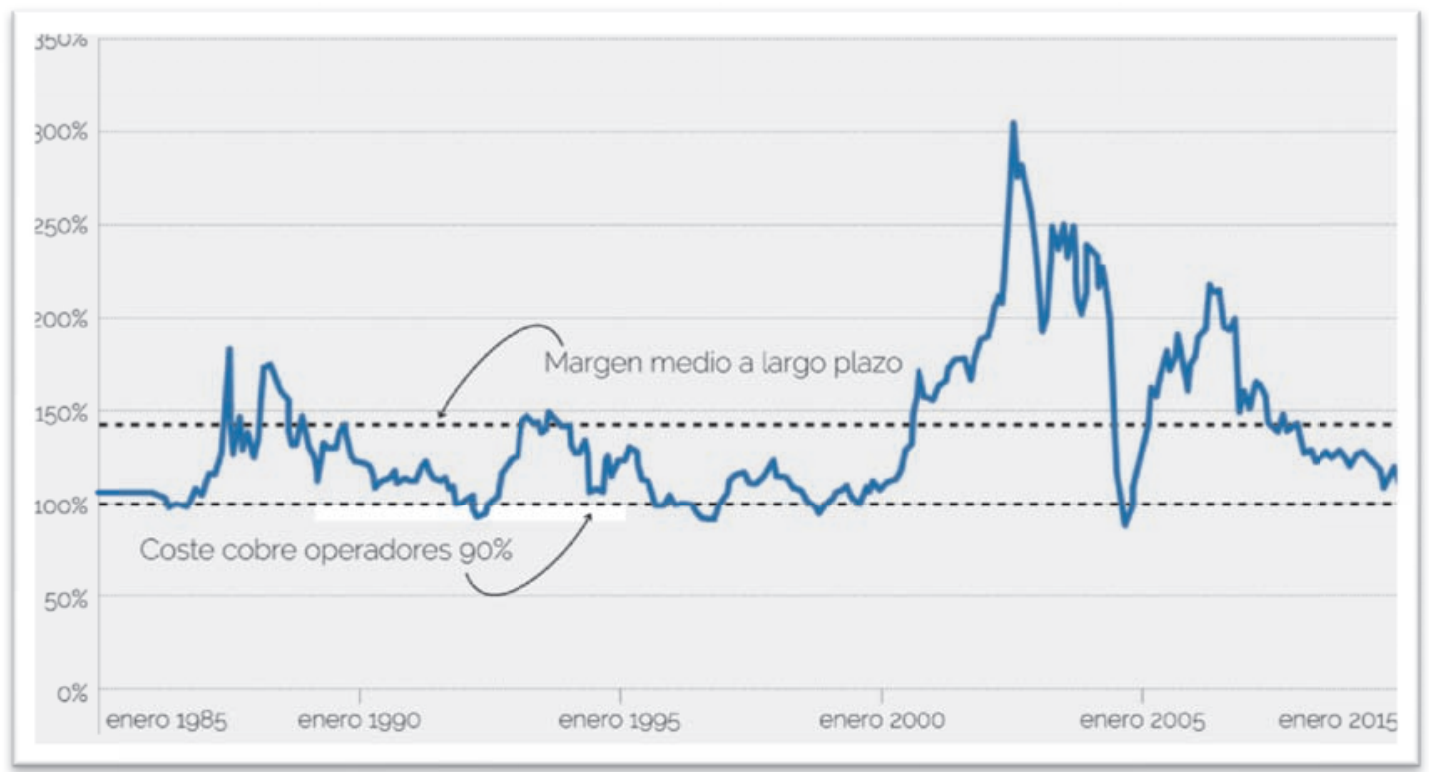

Figura 13. Influencia del coste de operación en el precio del cobre. En primer período el precio del cobre destruía la oferta (azValor). Figure 13. Consequences of operating cost in the copper price. The price of copper destroyed the offer (azValor).

Por tanto, tras analizar todo lo anterior, Antofagasta resultó ser una empresa con un crecimiento en la valoración de la acción del $150 \%$ desde enero del 2016 (Fig. 14). El estudio determinó sucesivas órdenes de compra de acciones en 2015, en valores mínimos, y su venta sucesiva ya entrado el ciclo alcista, en 2017, con todavía recorrido para seguir subiendo, pero alcanzando ya una rentabilidad del $100 \%$.

\section{Conclusiones}

En el análisis de una inversión en activos minerales, el conocimiento del entorno en dónde se mueve su actividad, puede resultar clave para el éxito de la operación a corto y medio plazo. Así, el sentido de la evolución de los precios de los metales debe apoyarse en el buen conocimiento de los factores que los condi- 


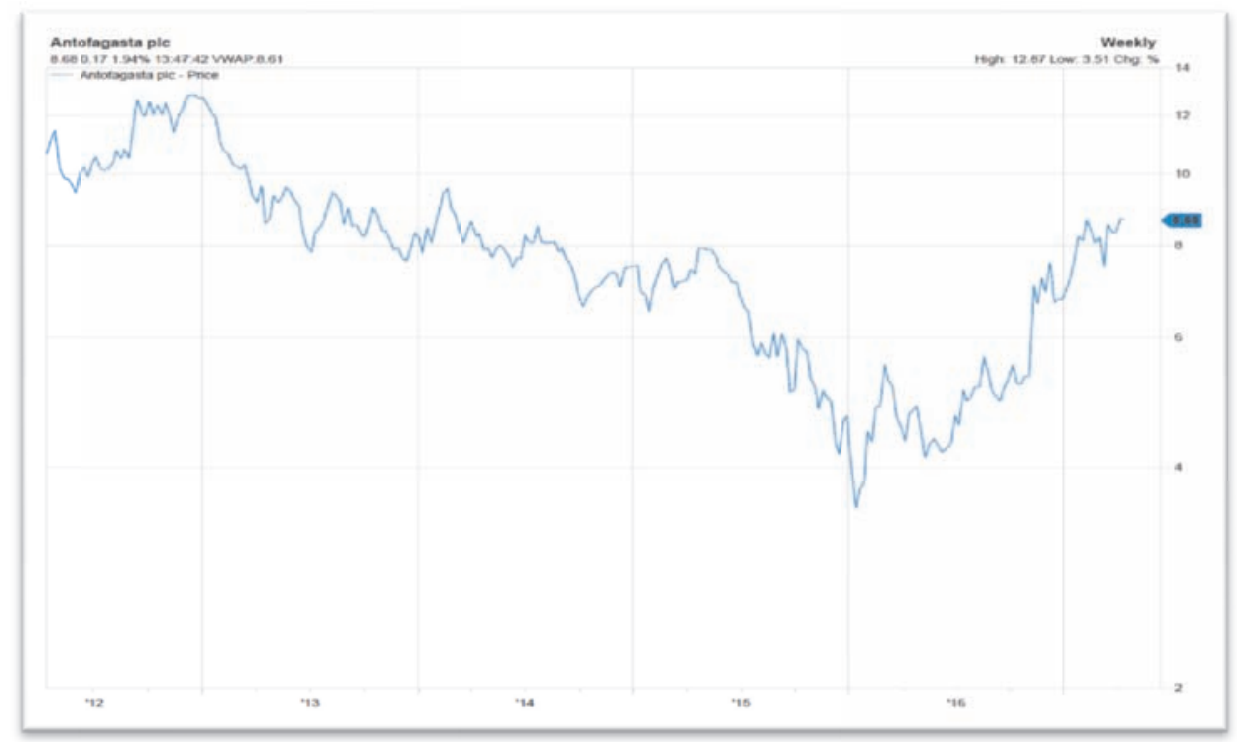

Figura 14. Precio de las acciones de Antofagasta (Facset). Figure 14. Price of Antofagasta shares (Facset).

cionan. Como ejemplo, los proyectos de explotación minera se apoyan en una realidad, en principio geológica, que también se puede expresar como su clasificación por modelos genéticos. Sin embargo, el sentido del modelo, sin darnos cuenta, viene a reflejar, no solo la génesis del depósito, sino que también, asume su sentido económico, técnico y productivo. De esta manera, como ejemplo, sobre un pórfido de cobre, al aceptar el término, también se aceptan sus condiciones genéricas de muy bajo ratio de desmonte, forma más o menos esferoide, ley baja, conocimiento o dominio metalúrgico de su concentración, intervalo de riqueza sus concentrados y varios aspectos más.

Para cerrar el ciclo y tal como se ha reflejado en el ejemplo anterior, la realidad del entorno del proyecto se enmarca en el conocimiento, tanto de los factores físicos como de la envoltura económico-financiera que acompaña al proyecto minero.

\section{Referencias}

Antofagasta PLC. 2017. www.antofagasta.co.uk Bloomberg, 2017. Electric-Car Revolution Shakes Up the Biggest Metals Market. https://www.bloomberg.com/ news/articles/2017-08-02/electric-car-revolution-is-shaking-up-the-biggest-metals-markets.
De laTorre, L., Espi J.A. 2017. Ciclo de Conferencias GERRM, Las razones geológicas de la actual minería del cobre (en publicación en este número BGM)

DeYoung J.H. 1981.The Lasky cumulative tonnage-grade relationship; a reexamination. Economic Geology, 76 (5): 1067-1080.

E\&MJ 2014, E\&MJ's Annual Survey of Global Metal-mining Investment. January 2014. https://www.e-mj.com/features/e-mj-s-annual-survey-of-global-metal-mining-investment/

Ivanhoe Mine, 2018. Presentation https://www.ivanhoemines.com/investors /presentations/

Larson, V., Ericsson M. 2014. Annual Survey of Global Metal-mining Investment, Engineering Mining Journal.

World Bank. 2017. Commodities Market Outlook, Oct.2017

Wood Mackenzie. 2014. Metal Costs Benchmarking 2014, Q4.

LME. 2017. https://www.Ime.com

Manteca, J.I. 1993. De la modelización del yacimiento a la producción mineral diaria. Universidad Complutense de Madrid. Tesis Doctoral. Madrid.

Serengeti Resources. 2017. Kwanika Project, https:// www.serengetiresources.com

Southern Copper. http://www.southerncoppercorp.com

Tematicas. 2018. https://tematicas.org/

US Department of Commerce Bureau of Labor Statistics. Wood Mckenze. https://www.bls.gov/

Wellmer F.W. and Drobe M. 2018. Quick Estimation of Economics of Exploration Projects--Rules-of-Thumb of Mine Capacity Revisited-the Input for Estimating Capital and Operating Costs. En este númeo BGM.

Recibido: diciembre 2017

Revisado: febrero 2018

Aceptado: junio 2018

Publicado: marzo 2019 